\title{
FEASIBILITY OF DETECTING MYOCARDIAL ISCHAEMIA USING THREE-DIMENSIONAL SPECKLE-TRACKING ECHOCARDIOGRAPHY
}

doi:10.1136/heartjnl-2012-302920k.2

Li Chen, Li Chun-mei, Bai Wen-juan, Zhang Xiao-ling, Tang Hong, Rao Li. West China Hospital of Sichuan University

Objectives Strain imaging provides objective quantification of myocardium deformation, thus is a useful tool to detecting regional wall motion abnormalities caused by myocardial ischaemia. With the technologic advancement in real-time three-dimensional echocardiography, the three-dimensional speckle tracking echocardiography (3DSTE) based on high quality volume image has been developed very recently. We aim to investigate the value of the novel 3DSTE in detecting myocardial ischaemia.

Methods Forty-six patients with acute coronary syndrome were included and received coronary angiography (CAG). Patients were divided into myocardial ischaemia group (with $>70 \%$ coronary stenosis in at least one main branch) and control group (without $>70 \%$ coronary stenosis) according to the results of (CAG). The conventional and 3D echocardiography was performed using a Vivid E9 ultrasound diagnosis platform (GE Vingmed Ultrasound, Chicago, US). Echo parameters were compared between groups. Ischaemic myocardium segments were defined as territory of coronary arteries with $>70 \%$ luminal stenosis. ROC curve analysis was performed to evaluate the accuracy of wall motion score (WMS) and strain parameters for detecting ischaemic segments. 


\section{ABSTRACTS}

Results CAG results showed 26 out of 46 patients had $>70 \%$ coronary artery stenosis. Speckle tracking were achieved in $94.8 \%$ (741/782) segments, with a total of 102 segments classified as ischaemic. The myocardial ischaemia group had significantly lower LVEF $(66.1 \% \pm 6.6 \%$ vs $49.8 \% \pm 12.0 \%, \mathrm{p}<0.001)$ and higher WMS index $(1.01 \pm 0.03$ vs $1.24 \pm 0.32, p=0.003)$ compared with control group. As for the strain parameters, global longitudinal strain (LS), circumferential strain (CS), radial strain (RS) and area strain (AS) were all impaired in myocardial ischaemia group (Global LS: $-19.1 \% \pm 3.1 \%$ vs $-15.1 \% \pm 3.2 \%$, p $<0.001$; Global CS: $-18.3 \%$ $\pm 2.5 \%$ vs $-15.0 \% \pm 3.0 \%$, p $<0.001$; Global RS: $53.7 \% \pm 10.3 \%$ vs $41.2 \% \pm 9.6 \%$, $p<0.001$; Global AS: $-33.1 \% \pm 4.1 \%$ vs $-27.0 \%$ $\pm 5.2 \%, \mathrm{p}<0.001 ;)$. ROC curve analysis showed the sensitivity and specificity of conventional WMS to detect myocardial ischaemia is $54.9 \%$ and $93.3 \%$, with an area under curve (AUC) of 0.744 . All four strain parameters measured by 3DSTE were proved useful in detecting myocardial ischemia (LS: sensitivity $67.6 \%$, specificity $78.7 \%$, AUC 0.827 ; CS: sensitivity $72.5 \%$, specificity $78.2 \%$, AUC 0.827; RS: sensitivity $81.4 \%$, specificity $82.6 \%$, AUC 0.886 ; AS: sensitivity $83.3 \%$, specificity $84.5 \%$, AUC 0.905 ), with the novel AS yield the highest accuracy.

Conclusions The novel AS derived by 3DSTE is a very accurate and reproducible index for detecting regional wall motion abnormalities caused by myocardial ischaemia. 